\title{
CIRUGÍA MINIMAMENTE INVASIVA EN UROLOGÍA PEDIÁTRICA DENTRO DE UN SERVICIO DE UROLOGÍA GENERAL
}

\author{
Jorge Subirá Ríos, José Manuel Sánchez Zalabardo, Joaquín Navarro Gil, José Ignacio Hijazo \\ Conejos, Jesús García-Magariño Alonso, David García Calero, José Antonio López López y \\ José Gabriel Valdivia Uría.
}

Servicio de Urología. Hospital Clínico Universiario Lozano Blesa. Zaragoza. España.

\begin{abstract}
Resumen.- OBJETIVO: Los avances en cirugía minimamente invasiva en urología en los últimos años han permitido la paulatina y constante introducción de la endourología y laparoscopia en pacientes pediátricos. Realizamos una revisión de nuestra experiencia, como hospital general, de la cirugía minimamente invasiva realizada en los últimos 10 años en pacientes pediátricos.

MÉTODOS: Se analizan retrospectivamente las intervenciones en el ámbito de la endourología y laparoscopia realizadas durante el periodo entre 1997 y 2007 en niños de hasta 16 años, recogiendo datos sobre la edad y sexo de los pacientes, tipo de patologías, técnicas empleadas, anestesia realizada e incidencias perioperatorias.
\end{abstract}

RESULTADOS: Se realizaron 72 intervenciones quirúrgicas en pacientes con un rango de edad entre 28 días y 16 años y una media de 6,8 años. El 56\% son varones y el 44\% niñas. 28 intervenciones por Reflujo Vesico Renal (RVR) (38,8\%), 17 intervenciones por patología litiásica $(23,6 \%)$ de las cuales 4 fueron cistolitotricias, 9 ureterorrenolitotricia, 1 nefrolitectomía percutánea (NLP) pura y 3 mixtas. 9 intervenciones sobre ureteroceles $(12,5 \%), 7$ por obstrucciones uretrales $(9,7 \%), 3$ criptorquidias diagnósticas laparoscópicas (4, 1\%), 2 intervenciones mediante laparoscopia por patología quistita $(2,7 \%)$ y otras 2 laparoscopias para realización de biopsia renal $(2,7 \%)$ y un síndrome de la unión pieloureteral intervenido mediante laparoscopia. Un caso de nefrostomía percutánea en decúbito supino de urgencia tras pieloplastia abierta con posterior intervención mediante resección percutáneo de granuloma. Un caso de inyección de toxina botulínica en detrusor.

CONCLUSIONES: La consolidación en nuestro servicio de la endourología, y mas recientemente la laparoscopia infantil, contribuyendo a mejorar la calidad asistencial en el paciente pediátrico lográndose gracias a un conocimiento previo en técnicas endourológicas generales y de unos medios técnicos y humanos adecuados.

Palabras clave: Endourología. Cirugía pediátrica. Laparoscopia.

Summary.- OBJECTIVES: The advances of minimaIly invasive surgery in urology over the last years have enabled a progressive and constant implementation of endourology and laparoscopy in pediatric patients. We perform a review of our experience, as a general 
hospital, with minimally invasive surgery performed in pediatric patients over the last ten years.

METHODS: We retrospectively analyzed the endourological and laparoscopic operations performed between 1997 and 2007 in children up to the age of 16 years, collecting data about patient's age and gender, type of disease, techniques, anesthesia, and perioperative events.

RESULTS: seventy-two surgical operations were performed in patients with an age range between 28 days and 16 years, with a mean age of 6.8 years. $56 \%$ of the patients were boys and $44 \%$ girls. Indications for surgery was vesicoureteral reflux (VUR) in 28 cases (38.8\%); lithiasis 17 cases (23.6\%) which were distributed in 4 cystolithotripsies, 9 ureterorenoscopy with lithotripsy, one pure percutaneous nephrolithotomy and three mixed; ureterocele 9 cases (12.5\%); urethral obstruction 7 cases (9.7\%); 3 diagnostic laparoscopies for cryptorchidism (4.1\%), 2 laparoscopic procedures for cystic pathology $(2.7 \%)$, another 2 laparoscopic renal biopsies $(2.7 \%)$, and one laparoscopic repair of a ureteropyelic junction syndrome; 1 case of emergency percutaneous nephrostomy in the supine position after open pyeloplasty with subsequent reoperation with percutaneous resection of a granuloma; and 1 case of botulin toxin injection into the detrusor muscle.

CONCLUSIONS: The consolidation of pediatric endourology in our department, and more recently laparoscopy, has contributed to improve the quality of care in pediatric patients; it has been achieved thanks to our previous know-how in general endourological techniques and the existence of adequate technical and human resources.

Keywords: Endourology. Pediatric surgery. Laparoscopy.

\section{INTRODUCCIÓN}

Las indicaciones de cirugía minimamente invasiva en adultos están en la actualidad bien definidas incluido sus ventajas frente a la cirugía abierta en cuanto a una disminución de la morbi-mortalidad posoperatoria, de la estancia hospitalaria y del tiempo para volver a realizar una actividad normal junto con unos mejores resultados estéticos $(1,2)$.

La aplicación de técnicas endourológicas en niños se vió retrasada debido a la dificultad de adaptar el instrumental a las características anatómicas de los pacientes pediátricos.

Actualmente hay material para cirugía minimamente invasiva específico para la edad pediátrica gracias a la plena instauración y continuo desarrollo de estas técnicas en los pacientes urológicos (2-4).

La endoscopia progresó con rapidez desde la cistoscopia diagnóstica y la ablación de la válvula transuretral hasta aplicaciones variadas, comparables con todas las técnicas endourológicas que se utilizan en los adultos. Esta expansión ofrece a los pacientes pediátricos las ventajas de métodos menos invasivos para tratar diversas patologías urológicas como cálculos, estenosis, riñones no funcionantes, reflujos vesico renales aunque también expone a los urólogos a la exigencia de adaptar estas técnicas a pacientes más pequeños con distintas necesidades clínicas (4-8).

En nuestro medio los pacientes pediátricos con patología urológica son tratados tanto en unidades de urología pediátrica, como en servicios de urología general. Este último caso presenta el inconveniente de la falta de especialización y dispersión de los casos (9).

Presentamos nuestra experiencia en el tratamiento endourológico de los pacientes pediátricos integrado en un Servicio de Urología General con amplia tradición en cirugía mínimamente invasiva.

\section{PRESENTACIÓN}

En el Hospital Clínico Universitario Lozano Blesa (HCU) la Urología Pediátrica se realiza dentro del servicio de urología general y en relación estrecha con el servicio de pediatría en cuyas camas los pacientes son ingresados hasta los 16 años de edad.

Este trabajo pretende realizar una revisión del papel que juega en la actualidad la cirugía minimamente invasiva en Urología pediátrica en nuestro servicio como ejemplo de unidad pediátrica incluida dentro de un servicio de Urología general.

\section{MATERIAL Y MÉTODOS}

Se realiza un estudio retrospectivo de todos aquellos pacientes, que de manera programada, han sido intervenidos quirúrgicamente mediante procedimientos endourológicos y/o laparoscópicos ya sea para fines diagnósticos como terapéuticos entre 1997 y 2007 en el Servicio de Urología del Hospital Clínico Universitario Lozano Blesa de Zaragoza.

Se revisan las historias clínicas y protocolos operatorios en pacientes de 0 a 16 años, recogiendo 
los datos demográficos, clínicos, operatorios y evolutivos de las distintas patologías pediátricas.

Se analizan dichos datos, comparándolos si procede con la misma patología en edad adulta para valorar si existe alguna diferencia entre ambos grupos.

\section{RESULTADOS}

En el periodo comprendido entre 1997 y 2007 se realizaron 72 intervenciones de cirugía minimamente invasiva en 64 pacientes.

La edad media fue de 8,4 años con un rango entre 28 días y 16 años.

De los 64 pacientes, 35 (54\%) fueron varones y $29(46 \%)$ niñas.

De las 72 intervenciones, $28(38,8 \%)$ fueron por reflujo vesico renal (RVR), 17 intervenciones $(23,6 \%)$ fueron por patología litiásica, 9 por diagnóstico de ureterocele $(12,5 \%), 5$ válvulas uretrales $(6,9 \%), 3$ diagnósticos de criptorquidias abdominales $(4,1 \%), 1$ síndrome de la unión $(1,3 \%), 2$ intervenciones por patología quística/tumoral $(2,7 \%)$, 2 estudios renales $(2,7 \%)$ y 2 estenosis uretrales $(2,7 \%)$

Para el tratamiento del RVR se empleó en 17 ocasiones inyección con Teflón $(60,7 \%)$ y en 6 ocasiones inyección de Coaptite (21,4\%), 3 con Deflux $(10,7 \%)$ y en 2 ocasiones con microesferas de carbono $(7,2 \%)$.

En el tratamiento de patología litiásica (17 intervenciones) se realizaron 4 cistolitotricias $(23,5 \%)$, 9 ureterolitotricia $(52,9 \%), 1$ nefrolitectomía percutánea y 3 intervenciones $(17,6 \%)$ mediante abordaje mixto: percutaneo y ureterorrenoscopia.

De los 9 casos de ureterocele en 8 se realizó apertura con asa y en 1 caso se empleó láser Holmium.

Nueve fueron las intervenciones en las que se empleó abordaje laparoscópico: En una ocasión para la resolución de síndrome de la unión.

Dos nefrectomías laparoscópicas, una por tumor Wilms y en otra ocasión por nefroma quístico multilocular.

En 3 casos se realizó el diagnóstico de criptorquidia abdominal mediante uso de laparoscopia.
Se empleó en 2 ocasiones la vía laparoscópica para la realización de biopsia renal y en una ocasión para la resección percutánea de granuloma por cuerpo extraño(sutura) tras pieloplastia abierta.

El tipo de anestesia empleada fue en $67 \mathrm{ca}-$ sos $(93 \%)$ general, en 3 casos intradural $(4,1 \%)$ y en 2 ocasiones $(2,7 \%)$ se empleó sedación IV.

La única complicación acontecida fue la imposibilidad de finalizar una NLP en un riñón en herradura precisando reconversión a cirugía abierta.

\section{DISCUSIÓN}

La posibilidad de contar con unidades especializadas en Urología Infantil tiene grandes ventajas, ya que supone un mayor número de pacientes con una atención más dirigida a las patologías propias de la edad pediátrica y la presencia en muchos casos de equipos multidisciplinares, con cirujanos infantiles y urólogos dedicados en exclusiva a estos pacientes. Esto permite una mayor experiencia tanto clínica como quirúrgica, difícil de conseguir en un Servicio de Urología General.

Por otra parte, la generalización de la Endourología y la laparoscopia en muchos Servicios de Urología General, como en nuestro caso, permite un adiestramiento y manejo casi diario de patologías muy poco frecuentes en niños, pero muy prevalentes en adultos, por lo que el abordaje mínimamente invasivo utilizado en los niños no difiere de la práctica clínica habitual evitando la curva de aprendizaje obligada en un servicio exclusivamente pediátrico.

No hemos encontrado especial dificultad en las técnicas endourológicas en los niños, ya que además presentan ciertas ventajas en comparación a la vía urinaria del adulto.

En los niños, los tejidos son más laxos y manejables, que permiten por ejemplo exteriorizar la unión pieloureteral a través de uno de los trocares para realizar la anastomosis pieloureteral. Este procedimiento no se lleva a cabo en nuestro servicio, realizando la pieloplastia completa por vía laparoscópica pero es una alternativa en casos de menor experiencia con sutura laparoscópica.

También suele existir una mayor complacencia del uréter, con gran facilidad tanto para la introducción de ureteroscopios infantiles como para la expulsión de fragmentos litiásicos tras una litotricia extracorpórea o endoscópica. 
Mayor capacidad de regeneración, que permite evitar la colocación de tutores ureterales o nefrostomías tras cirugía laparoscópica o abierta de la estenosis pieloureteral.

Desde hace 23 años en nuestro servicio venimos realizando el acceso percutaneo al riñón en decúbito supino con demostrada eficacia y seguridad, presentando ventajas con respecto a la posición en prono. Todos los accesos percútanlos realizados en pacientes pediátricos se han realizado mediante este abordaje sin hallarse complicaciones en cuanto al acceso.

\section{CONCLUSIONES}

La consolidación en nuestro servicio de la endourología, y más recientemente la laparoscopia infantil, contribuye a mejorar la calidad asistencial en el paciente pediátrico. Esto se logra gracias a un conocimiento previo en técnicas endourológicas generales y de unos medios técnicos y humanos adecuados.

Nuestra actividad endourológica diaria nos ha permitido abordar la patología infantil sin precisar un gran número de pacientes, pudiendo ofrecer a estos niños el tratamiento menos invasivo para solucionar su enfermedad.

Recomendamos el acceso percutaneo en decúbito supino dado su eficacia y seguridad demostrada en adultos y que es reproducible en edad pediátrica.

\section{BIBLIOGRAFÍA y LECTURAS RECOMENDADAS (*lectura de interés $y^{* *}$ lectura fundamental)}

**1. VALDIVIA URÍA, J.G.: "Cirugía endoscópica en urología". Actas Urol. Esp., 26: 552, 2002.

2. DOCIMO, S.G.: "Pediatric endourology". J. Urol., 162: 1731, 1999.

*3. DOCIMO, S.G.; PETERS CRAIG, A.: "Campbell Urology" Ed. Panamericana, 2791-2821, Buenos Aires, 2004.

**4. VALDIVIA URÍA, J.G.; LÓPEZ LÓPEZ, J.A. y cols.: "Cirugía Minimanente Invasiva en Urología" 205-210, Ed. Ene Ediciones, Madrid, 1993.

5. FARKAS, A.; MORIEL, A.: "Endoscopic correction of vesicoureteral reflux: Our experience with 115 ureters". J. Urol., 144: 534, 1990.

6. KAPLAN, W.; DALTON, D.; FIRLIT C.: "The endoscopic correction of reflux by polytetrafluoroethylene injection". J. Urol., 138: 953, 1987.

*7. MATOUSCHEK, E.: "Sobre un nuevo concepto para el tratamiento reflujo vesicoureteral". Arch. Esp. Urol., 34: 385, 1981.

8. SCHULMAN, C.C.; SIMON, J.; PAMART. y cols.: "Endoscopic treatment of vesicoureteral reflux in children". J. Urol., 138: 950, 1987.

**9. MINGUÉLEZ, C.; GARAT, J.M.: "Presente y futuro de la urología pediática". Cir. Pediatr., 16: 157, 2003. 